Virginia Commonwealth University vCU Scholars Compass

\title{
Operative and Nonoperative Management of Blunt Liver Injuries
}

Jill Peysha

Virginia Commonwealth University

Paula Ferrada

Virginia Commonwealth University,pferrada@mcvh-vcu.edu

Follow this and additional works at: http://scholarscompass.vcu.edu/surgery_pubs

Part of the Surgery Commons

(C) Springer International Publishing AG 2017

\section{Downloaded from}

http://scholarscompass.vcu.edu/surgery_pubs/47

This Article is brought to you for free and open access by the Dept. of Surgery at VCU Scholars Compass. It has been accepted for inclusion in Surgery Publications by an authorized administrator of VCU Scholars Compass. For more information, please contact libcompass@vcu.edu. 


\title{
Operative and Nonoperative Management of Blunt Liver Injuries
}

\author{
Jill Peysha $^{1,2} \cdot$ Paula Ferrada $^{1,2}$
}

Published online: 6 February 2017

(C) Springer International Publishing AG 2017

\begin{abstract}
Purpose of Review The purpose of this review is to discuss the indications for operative and nonoperative management in patients with blunt liver injuries.

Recent Findings Over the past several years, research has begun to show that nonoperative management in blunt liver injuries is feasible and has favorable outcomes over immediate operations in patients who are hemodynamically stable. This includes highgrade injuries who were previously taken to the operating room, in the absence of peritoneal signs or instability, for washout and packing. This trend in management is likely multifactorial and includes improved quality of critical care medicine, advances in imaging to accurately define the injury and trend changes, and other interventional techniques such as embolization, percutaneous drainage and endoscopy. The mainstay of treatment for hemodynamically unstable patients remains operative.

Summary This article will provide current recommendations for operative and nonoperative management strategies in patients with blunt liver injuries, taking into account initial clinical picture and resources available.
\end{abstract}

Keywords Blunt liver injury · Operative management . Nonoperative management $\cdot$ Embolization $\cdot$ Failure of nonoperative management

This article is part of the Topical Collection on Blunt Abdominal Trauma

Paula Ferrada

pferrada@mcvh-vcu.edu

1 Critical Care and Emergency Surgery, VCU Surgery Trauma, West Hospital, 15th Floor, East Wing, 1200 E. Broad St., PO Box 980454, Richmond, VA 23298-0454, USA

2 Department of Surgery, Virginia Commonwealth University, Richmond, VA 23298, USA

\section{Introduction}

The liver is the most frequently injured organ in blunt abdominal trauma and therefore necessitates timely and effective management when encountered [1]. Initially, treatment for liver injuries included an immediate exploratory laparotomy to evaluate the extent of injury and repair as needed. This option has been shown to increase morbidity and mortality in the trauma population, and trials of nonoperative management were initiated $[2 \cdot \bullet]$. Now with the advent of improved computed tomography which can assess injury severity, the presence of hemoperitoneum, active extravasation, and additional abdominal injuries, as well as provide accurate follow up studies to assess change, nonoperative management is possible and provides improved outcomes. In fact, nonoperative management has been shown to be successful in $>90 \%$ cases [3]. Nonoperative management has also had similar results in pediatric patients.

\section{Classification of Liver Injury}

The classification of liver injury is based on the severity of the injury, as set forth by the American Association of Surgery for Trauma [4•].

- Grade I-hematoma: subcapsular $<10 \%$ surface area; laceration: capsular tear $<1 \mathrm{~cm}$ parenchymal depth (Fig. 1)

- Grade II - hematoma: subcapsular 10 to $50 \%$ surface area, intraparenchymal $<10 \mathrm{~cm}$ in diameter; laceration: capsular tear 1 to $3 \mathrm{~cm}$ parenchymal depth, $<10 \mathrm{~cm}$ in length (Fig. 2)

- Grade III-hematoma: subcapsular $>50 \%$ of surface area or ruptured subcapsular or parenchymal hematoma; 


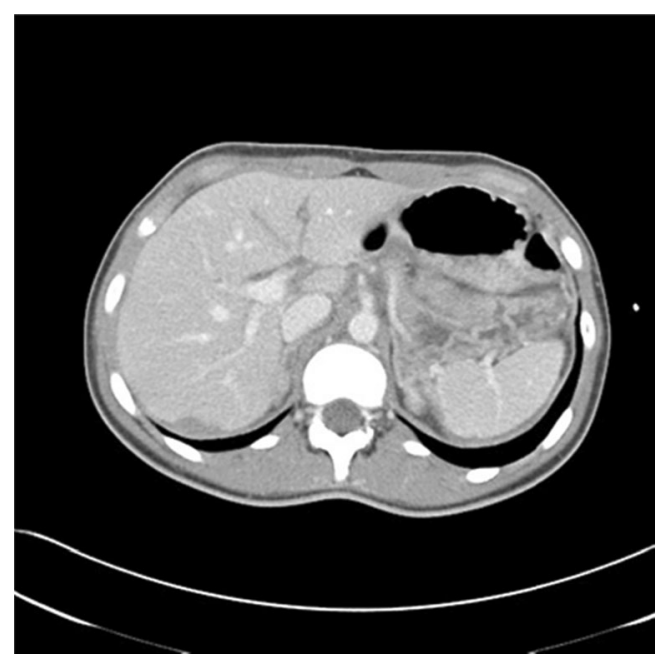

Fig. 1 Grade I-hematoma. Figure courtesy of RMH Core Conditions, Radiopaedia.org, rID: 34255

intraparenchymal hematoma $>10 \mathrm{~cm}$ or expanding; laceration $>3 \mathrm{~cm}$ in depth (Fig. 3)

- Grade IV-laceration: parenchymal disruption involving 25 to $75 \%$ of a hepatic lobe or 1 to 3 Couinaud segments (Fig. 4)

- Grade V-laceration: parenchymal disruption of $>75 \%$ of a hepatic lobe, $>3$ Couinaud segments within a single lobe; vascular: juxtahepatic venous injuries (Fig. 5)

- Grade VI-hepatic avulsion

While a higher grade has a higher potential for failure of nonoperative management simply given its increased severity of injury, the grade itself does not dictate operative vs nonoperative management, but rather the hemodynamic appearance of the patient.

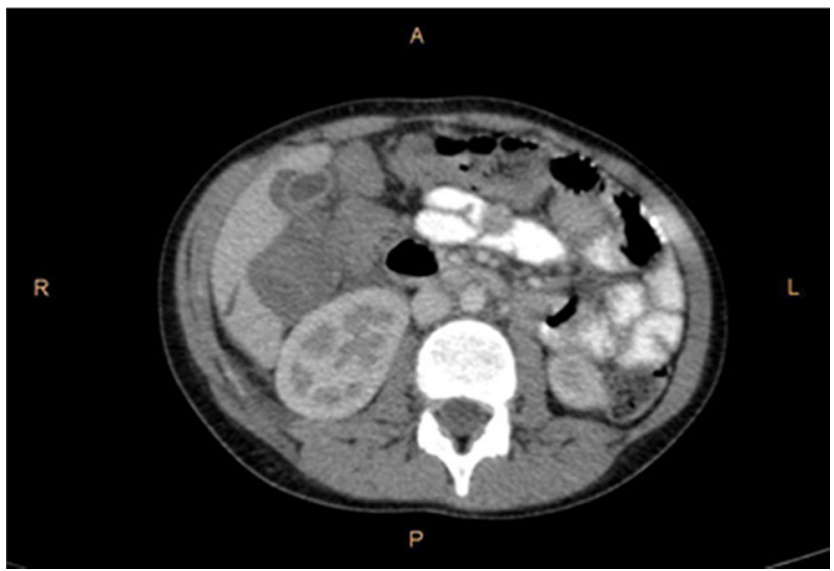

Fig. 2 Grade II-hematoma. Figure courtesy of Dr Ahmed Abd Rabou, Radiopaedia.org, rID: 22841

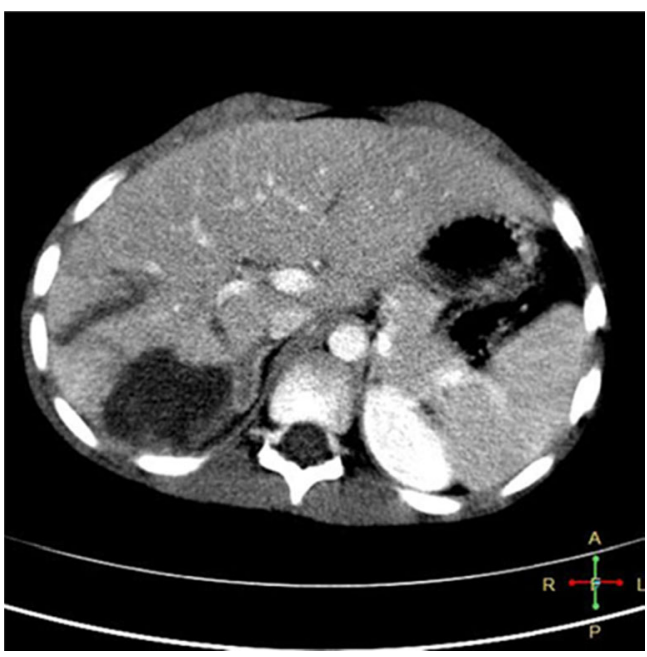

Fig. 3 Grade III-hematoma. Figure courtesy of Dr Ahmed Abd Rabou, Radiopaedia.org, rID: 24572

\section{Operative Management}

Operative management is mandated in patients with hemodynamic instability, peritonitis, failure of alternative nonsurgical interventions, or evidence of additional injury to an intraabdominal structure that requires operative examination and repair. Thorough knowledge of anatomy and surgical techniques are useful during an intra-abdominal exploration and essential to proper treatment $[6 \bullet \bullet]$.

\section{Intra-abdominal Exploration}

After entering the abdomen, the first step should be packing of the four quadrants to provide compressive therapy and allow

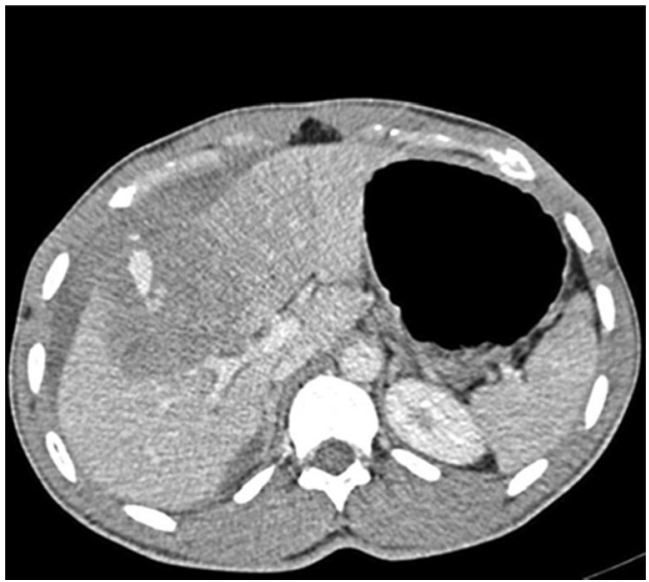

Fig. 4 Grade IV-laceration. Figure courtesy of Dr Ian Bickle, Radiopaedia.org, rID: 19226 


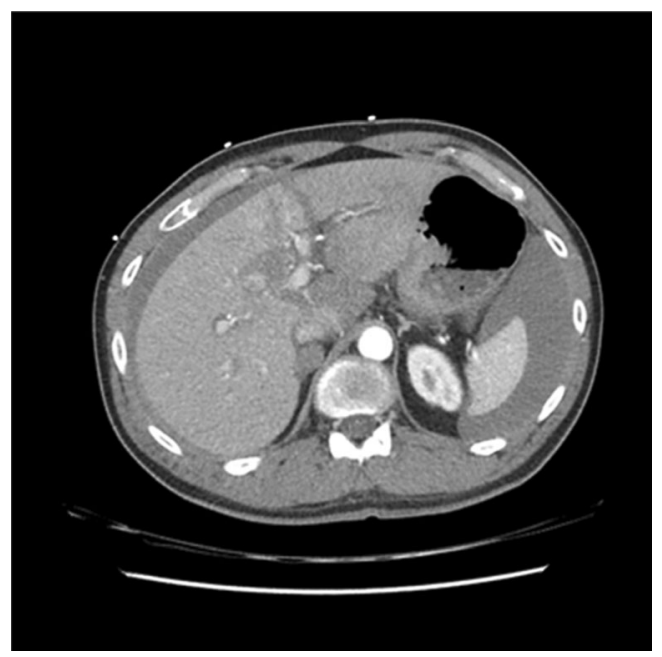

Fig. 5 Grade V-laceration. Figure courtesy of Dr Andrew Dixon, Radiopaedia.org, rID: 32502

for resuscitation and a clear operative field. The packs should be removed from the lower quadrants first and ending with the right upper quadrant where injury is expected. Splenectomy can be performed at this time if a concurrent splenic injury is identified [19]. The liver should then be addressed. Finger fracture can be employed in which the liver parenchyma is crushed between the thumb and one finger which allows individual vessels and bile ducts to be identified and ligated [7]. The vessels are ligated with absorbable suture and hemostasis of larger lacerations can be obtained with sutures and patches of omentum [20]. Liver stapling allows for transection and ligation of liver panenchyma, arteries, veins, and ducts in a quick, safe, and efficient manner and is useful in larger sections that require surgical excision [16]. The Pringle maneuver, which clamps the hepatoduodenal ligament and therefore the hepatic artery, portal vein, and common bile duct, can be performed for up to $1 \mathrm{~h}$ to limit blood flow through the liver and therefore more easily identify injuries [14]. If caval and hepatic vein injury is suspected, hepatic isolation can be performed during which a Pringle maneuver is performed followed by clamping of the inferior vena cava above and below the injury $[12,20]$. For more severe injuries of the cava when the patient is in shock, an atrio-caval shunt can be performed [8]. A $36 \mathrm{~F}$ chest tube or 9-mm ET tube is inserted into the right atrium and then into the inferior vena cava to create the shunt. This shunt when combined with the Pringle maneuver will usually control most bleeding. Because this technique is only used in very critical patients and because of the complexity of the procedure, the outcomes are not very favorable with a survival of about $20 \%$ [8]. In the event the patient is too unstable to continue with surgery and face potential blood loss, a damage control operation can be performed that requires extensive perihepatic packing to tamponade the liver with transfer to the ICU until a second look operative with definitive treatment. In the meantime, metabolic derangement should be corrected. In massive injuries where bleeding is unable to be controlled, the patient is coagulopathic, acidotic, and hypothermic, or if there is postoperative liver failure, hepatic transplantation can be considered; however, less than 20 transplants have been successful in the past 25 years [13].

\section{Nonoperative Management}

Nonoperative management is feasible in patients who are hemodynamically stable with no evidence of peritonitis on exam. Greater than ninety percent of patients with liver injuries are able to be managed in this way. Stable patients initially receive a CT scan which can classify the grade of injury and important factors such as active extravasation and concomitant injuries. In the absence of other injuries, the patient is taken to interventional radiology where an angiography with embolization can be performed and prevent operative ligation [5]. Endoscopic retrograde cholangiopancreatography (ERCP) can also be used for diagnosis and treatment of bile duct injuries [3].

\section{Complications of Nonoperative Management}

Conservative nonoperative management is not without risk and can lead to a range of complications. In fact, these complications can been seen any time the liver in injured, including after surgical intervention. One possibility is formation of biloma or persistent bile leaks from a ductal injury. Symptoms suspicious for leak include tachycardia and leukocytosis after resuscitation and are identified by repeat $\mathrm{CT}$ scan and treated with percutaneous drainage or ERCP [18]. Magnetic resonance cholangiopancreatography (MRCP) has limited use in the evaluation of blunt liver injury given its time requirement and non-therapeutic nature, especially when CT can provide quick and critical information. MRCP can be used to evaluate for bile leak in a stable patient before a therapeutic ERCP. MRCP is also an option for follow-up of a biliary injury in young or pregnant patients or those with renal disease or contrast dye allergy [21]. Hemobilia is another potential complication that can arise after hepatic trauma and is clinically noted with Quincke's triad of upper abdominal pain, upper gastrointestinal bleed, and jaundice. Treatment for hemobilia depends on the severity of bleeding and ranges from observation and self-resolution to embolization to surgical vessel ligation. Perihepatic abscesses can form which would also require percutaneous drainage and antibiotics. Hepatic necrosis is a more severe complication that occurs after hepatic injury in addition to ischemia usually from embolization [17]. Necrosis presents in a variety of ways depending on the extent of hepatic involvement, from abdominal pain and feeding intolerance to 
sepsis and multiorgan system failure [15]. While this devascularization can result in complete regeneration of hepatic parenchyma, surgical debridement may be warranted in more extreme cases when multiple lobes are involved or for fulminant liver failure [22].

\section{Failure of Nonoperative Management}

About $5-10 \%$ of blunt liver injury patients that were initially managed nonoperatively will fail therapy and require operative intervention [9]. Reasons for failure include hemodynamic instability, inability to embolize actively bleeding vessel, peritonitis, and initial misdiagnosis of injury severity [10]. Indicators that increase the chances of nonoperative failure include grade 4-5 injury, increased age, decreased GCS, moderate or large hemoperitoneum, synchronous splenic injury, and elevated Injury Severity Score. Failure was associated with lower levels of hemoglobin, longer ICU length of stay, longer overall length of stay, and higher number of transfusions; the last also predicts mortality [11].

\section{Conclusions}

Liver injuries after blunt force have typically been managed operatively with exploratory laparotomy, four quadrant packing, identifying the injured segment, and repair of hepatic injury by finger fracture, stapling, or hepatic isolation. This technique should continue to be used in cases of initial hemodynamic instability or if the patient decompensates at any point after the causative trauma. In more severe cases non-anatomical liver resection can be used to reach the bleeding vessel, and ligation under direct visualization Nonoperative management, however, has gained increased popularity in blunt liver traumas given its high success rates and decreased morbidity and mortality, even in high-grade injuries. Advances in imaging and other, less invasive procedures have allowed for modalities such as $\mathrm{CT}$, angiography, percutaneous drainage, and ERCP to be used in order to control bleeding, drain bile leaks and abscesses, and prevent an open operation. With a proper protocol that includes monitoring of vitals, labs, follow-up imaging, rapid identification of complications, and ability to perform operative intervention if necessary, nonoperative management of blunt liver injury is an effective and preferred method, regardless of the injury grade.

\section{Compliance with Ethical Standards}

Conflict of Interest Drs. Peysha and Ferrada declare no conflicts of interest relevant to this manuscript.

Human and Animal Rights and Informed Consent This article does not contain any studies with human or animal subjects performed by any of the authors.

\section{References}

Papers of particular interest, published recently, have been highlighted as:

- Of importance

- Of major importance

1. Jiang H, Wang J. Emergency strategies and trends in the management of liver trauma. Front Med. 2012;6:225. doi:10.1007/s11684012-0186-6.

2.• Raza M, Abbas Y, Devi V, Prasad KVS, Rizk KN, Nair PP. Non operative management of abdominal trauma - a 10 year review. World J Emerg Surg. 2013;8:14. doi:10.1186/1749-7922-8-14. This reference examines 10 years of patients with liver injuries and concludes that nonoperative management is effective in managing blunt liver injuries with proper monitoring and protocols.

3. Van der Wilden GM, Velmahos GC, Emhoff T, Brancato S, Adams C, Georgakis G, et al. Successful nonoperative management of the most severe blunt liver injuries. Arch Surg. 2012;147(5):423-8. doi:10.1001/archsurg.2012.147.

4. Moore EE, Cogbill TH, Jurkovich GJ, et al. Organ injury scaling V: spleen and liver. J Trauma. 1995;38(3):323-4. This reference provides the standard grading system to classify liver injuries.

5. Wahl WL, Ahrns KS, Brandt MM, Franklin GA, Taheri PA. The need for early angiographic embolization in blunt liver injuries. Journal of Trauma-Injury Infection \& Critical Care 2002:52(6).

6.• Ward J, Alarcon L, Peitzman B. Management of blunt liver injury: what is new? Eur J Trauma Emerg Surg. 2015;41:229. doi:10.1007/s00068-015-0521-0. This reference provides an overview of nonoperative imaging modalities and procedures to manage blunt liver injuries and surgical options when necessary.

7. Poon RTP. Current techniques of liver resection. HPB. 2007;9(3): 166-73. doi:10.1080/13651820701216182.

8. Clark JJ, Steinemann S, Lau JM. Use of an atriocaval shunt in a trauma patient: first reported case in Hawai'i. Hawaii Med J. 2010;69(2):47-8.

9. Hsieh TM, Tsai TC, Liang JL, Lin CC. Non-operative management attempted for selective high grade blunt hepatosplenic trauma is a feasible strategy. World J Emerg Surg. 2014;9:15. doi:10.1186/17497922-9-51.

10. Peitzman AB, Ferrada P, Puyana JC. Surgical Infections 2009: 10(5):427-433. Doi:10.1089/sur.2009.021

11. Polanco PM, Brown JB, Puyana JC, Billiar TR, Peitman AB, Sperry $\mathrm{JL}$. The swinging pendulum: a national perspective of nonoperative management in severe blunt liver injury. J Trauma Acute Care Surg. 2013;75(4):590-5. doi:10.1097/TA.0b013e3182a53a3e.

12. Yoon W, Jeong YY, Kim JK, Seo JJ, Lim HS, Shin SS, et al. CT in blunt liver trauma. Radiographics 2005:25(1).

13. Latifi $\mathrm{R}$, Khalaf $\mathrm{H}$. Selective vascular isolation of the liver as part of initial damage control for grade 5 liver injuries: shouldn't we use it more frequently? Int J Surg Case Rep. 2015;6:292-5. doi:10.1016/j. ijscr.2014.12.021.

14. Ahmed N, Vernick JJ. Management of liver trauma in adults. J Emerg Trauma Shock. 2011;4(1):114-9. doi:10.4103/09742700.76846.

15. Abdelrahman $\mathrm{H}$, Ajaj A, Atique S, El-Menyar A, Al-Thani H. Conservative management of major liver necrosis after angioembolization in a patient with blunt trauma. Case Rep Surg. 2013;954050:1-4. doi:10.1155/2013/954050.

16. Yao DB, Wu SD. Application of stapling devices in liver surgery: current status and future prospects. World J Gastroenterol. 2016;22(31):7091-8. doi:10.3748/wjg.v22.i31.7091. 
17. Dabbs DN, Stein DM, Scalea RM. Major hepatic necrosis: a common complication after angioembolization for treatment of highgrade liver injuries. J Trauma. 2009;66(3):621-7. doi:10.1097/TA.0 b013e31819919f2.

18. Anand RJ, Ferrada PA, Darwin PE, Bochicchio GV, Scalea TM. Endoscopic retrograde cholangiopancreatography is an effective treatment for bile leak after severe liver trauma. J Trauma. 2011;71(2):480-5. doi:10.1097/TA.ob013e3181efc270.

19. Stassen NA, Bhullar I, Cheng JD, Crandall M, Friese R, Guillamondegui $\mathrm{O}$, et al. Blunt hepatic injury, selective nonoperative management of. J Trauma. 2012;73(5):S288-93.

20. Ordonez CA, Parra MW, Salamea JC, Puyana JC, Millan M, Badiel $\mathrm{M}$, et al. A comprehensive five-step surgical management approach to penetrating liver injuries that require complex repair. J Trauma Acute Care Surg. 2013;75(2):207-11. doi:10.1097/TA.0b13e31829 de5d1.

21. Kelly MD, Armstrong CP, Longstaff A. Characterization of biliary injury from blunt liver trauma by MRCP:case report. J TraumaInjury Infect Crit Care. 2008;64(5):1363-5. doi:10.1097/TA.0 b013e318075e84f.

22. Weng HL, Cai X, Yuan X, Liebe R, Dooley S, Li H, et al. Two sides of one coin: massive hepatic necrosis and progenitor cell-mediated regeneration in acute liver failure. Front Physiol. 2015;6:178. doi:10.3389/fphys.2015.00178. 